the fistulae. High-resolution intraoperative contrast-enhanced cone beam CT (CE-CBCT) allows visualization of arteriovenous vascular structures and their relationship with bony structures and the dura. We present 2 cases of complex DAVFs in which CE-CBCT, by clearly showing the fistulous point, allowed a targeted treatment that avoided unnecessary venous sacrifice and maintained/restored functionality of normal venous structures.

Materials and Methods In two patients who presented with complex DAVFs, DSA was insufficient in clarifying the anatomy of the shunt. CE-CBCT was obtained on a last generation biplane Neuroangiography system infusing a $69 \mathrm{~mL}$ bolus (30\% Visipaque 270 ) at a rate of $3 \mathrm{~mL} / \mathrm{second}$ into the main arterial feeder (22 cm FOV; 23 second 200-degree rotation). High-resolution volumetric data generated was reconstructed using an FBP algorithm and post-processed on a dedicated workstation for final treatment strategy.

Results A 90-year-old man with progressively slurred speech, had a petrosal/tentorial Cognard 4 DAVF fed by multiple left ECA feeders. CE-CBCT demonstrated an enlarged draining petrosal dural venous pouch draining in the basal vein of Rosenthal. Access route to the pouch was planned on CE-CBCT reconstructions (Image A, dotted line), allowing selective occlusion with coils and Onyx with complete resolution of the DAVF and preservation of normal deep venous structures. The patient's symptoms resolved shortly following the procedure.The second subject, a 49-year-old man presented with debilitating right-sided pulsatile tinnitus, blurred vision and headaches. A DSA demonstrated a complex, Cognard 1 fistula of the right sigmoid sinus with innumerable external carotid artery (ECA) feeders and loss of drainage functionality of the right transverse-sigmoid sinus. CE-CBCT showed feeders converging in a dural venous pouch adjacent to the postero-lateral wall of the sigmoid sinus (Image B, stars). Similarly to prior case, a CECBCT-planned microcathetarization was performed with targeted obliteration of the pouch with coils and Onyx followed by DAVF resolution and restoration of normal antegrade venous flow. Tinnitus resolved, but blurred vision and headaches persisted. Venous pressure measurements demonstrated a $10-\mathrm{mmHg}$ gradient across the narrowed sigmoid sinus. Venous stenting led to complete symptom resolution.

Conclusion High-resolution CE-CBCT allows identification of fistulous connections in complex DAVFs and supports an accurate and minimally-deconstructive treatment with sparing of normal venous structures.

Disclosures E. Orru: None. M. Marosfoi: None. N. Patel: None. A. Wakhloo: None.

\section{E-035 3D ROTATIONAL ANGIOGRAPHY OF PERSISTENT TRIGEMINAL ARTERY ANEURYSM CONNECTING TO THE SUPERIOR CEREBELLAR ARTERY}

${ }^{1} A$ Ravelo*, ${ }^{2} \mathrm{R}$ Williamson. ${ }^{1}$ Neurology, Allegheny Health Network, Pittsburgh, $P A_{i}$ ${ }^{2}$ Neurosurgery, Allegheny Health Network, Pittsburgh, PA

\subsection{6/neurintsurg-2020-SNIS.71}

Introduction Carotid-Basilar Anastomoses were original described in London in 1844 by Quain's. They are named using the cranial nerves and there are different persistent fetal anastomoses between the carotid and basilar artery including the primitive trigeminal, otic (acoustic), hypoglossal, and proatlantal intersegment arteries.

Persistent trigeminal artery (PTA) is an anatomic anomaly that result of failure the trigeminal artery to regress. PTA is the most common of these that persist into adulthood. Uchino et al, reported an incidence of $0.76 \%$ in a study done with MR angiographic.Luh et al, described in 1999, these fetal anastomoses emerge at the 4 to $5 \mathrm{~mm}$ embryonic stage, persist for approximately one week, and regress by the time of posterior communicating and vertebral arteries develop. The first to disappear is the optic artery, followed by the hypoglossal artery, the trigeminal artery, and then the pro-atlantal intersegmental artery.

We present a patient in whom a variant of PTA aneurysm was incidentally found terminating in the superior cerebellar artery (SCA), Saltzman classification type IIIa.

Methods A review of the medical literature using standard search engines was performed to locate articles regarding 


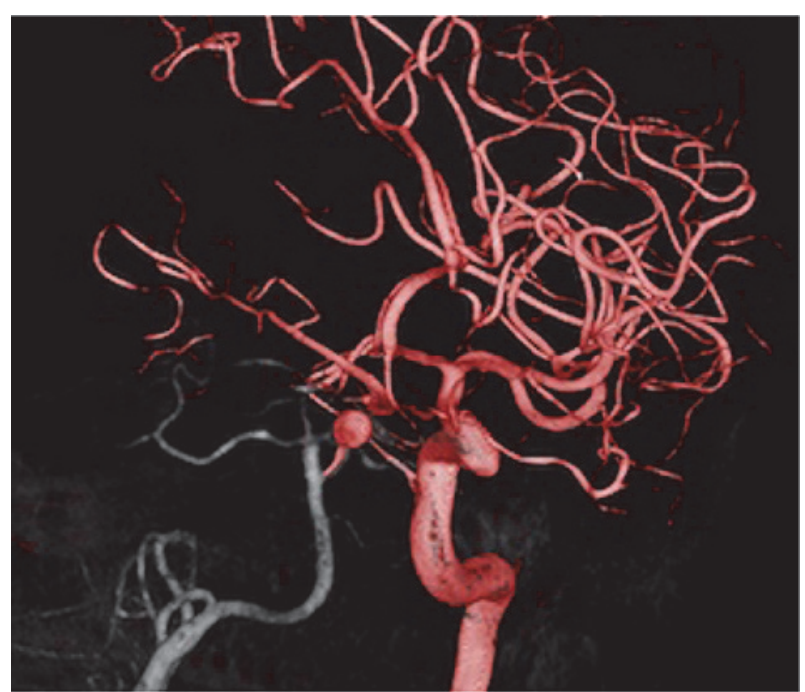

Abstract E-035 Figure 1

Carotid-Basilar anastomoses focusing in anatomic variants of the persistent trigeminal artery.

Results 61-year-old female who presented with quadriparesis after traumatic cervical spinal cord C4-C5 compression; requiring anterior cervical discectomy and fusion. During preoperatory work up, she was found to have a posterior circulation aneurysm in CT angiogram. Patient had a digital subtraction angiography (DSA) that showed a persistent trigeminal artery mid segment aneurysm $5.7 \mathrm{~mm} \times 5.5 \mathrm{~mm}$ in the dominant hemispheric branch and terminate in the SCA territory (Saltzman classification IIIa). After a DSA patient is schedule for endovascular treatment.

Conclusion Persistent trigeminal artery is one of the remnant fetal anastomoses that connect the cavernous segment of carotid artery to the basilar artery. PTA are classified according to angiography by Saltzman in 1959, he reviewed previous reports of PTA along with presenting eight cases and made an angiographic classification dividing in 3 groups. In addition, aneurysms of the PTA are extremely rare in the literature with previous cases reported. This case is unique because there is not report with a 3D rotational DSA of variant PTA aneurysm terminating in the SCA, Saltzman classification type IIIa.

Disclosures A. Ravelo: None. R. Williamson: None.

\section{E-036 NEW IN VITRO AVM MODEL WITH REALISTIC NIDUS FOR SIMULATION AND FLOW ANALYSIS}

${ }^{1} \mathrm{~N}$ Kaneko*, ${ }^{1} \mathrm{H}$ Ullman, ${ }^{1} \mathrm{~F}$ Ali, ${ }^{2} \mathrm{P}$ Berg, ${ }^{1} \mathrm{Y}$ Ooi, ${ }^{1} \mathrm{~S}$ Tateshima, ${ }^{1,3} \mathrm{G}$ Colby, ${ }^{4} \mathrm{Y}$ Komuro, ${ }^{1} \mathrm{P}$ Hu, ${ }^{1} \mathrm{~V}$ Szeder, ${ }^{1,4} \mathrm{M}$ Nour, ${ }^{1} \mathrm{~L}$ Guo, ${ }^{1} \mathrm{~A}$ Chien, ${ }^{1} \mathrm{~F}$ Vinuela, ${ }^{5} \mathrm{~S}$ Nemoto, ${ }^{4} \mathrm{~J}$ Hinman, ${ }^{1} \mathrm{G}$ Duckwiler, ${ }^{1} \mathrm{R}$ Jahan. ${ }^{1}$ Department of Radiological Sciences, UCLA, Los Angeles, CA; ${ }^{2}$ Research Campus STIMULATE, University of Magdeburg, Magdeburg, GERMANY; ${ }^{3}$ Department of Neurosurgery, UCLA, Los Angeles, CA; ${ }^{4}$ Department of Neurology, UCLA, Los Angeles, CA; ${ }^{5}$ Kanto Rosai Hospital, Kawasaki, JAPAN

\subsection{6/neurintsurg-2020-SNIS.72}

Background In vitro vascular models for brain aneurysms and acute stroke have been used for training, simulation and research purpose. However, the use of realistic in vitro models for arteriovenous malformation (AVM) have not been reported. Current in vitro AVM models analyzing the efficacy of embolic materials or flow conditions are limited due to a lack of realistic anatomical and dynamic features of complex nidus.

Materials and Methods 3D AVM nidus images were extracted and segmented from 3D rotational angiography from a patient. Additional artificial feeders and drainers were attached to the AVM nidus. The inner vascular mold was printed using a plastic 3D printer. The inner mold was coated with silicone and then removed with acetone, leaving a hollow AVM model. Injections of liuid embolic material and 4D flow MRI were performed using the 3D in vitro AVM model. Computational fluid dynamics (CFD) analysis was also performed to compare the flow volume and velocity to 4D flow MRI

Results The created in vitro AVM models had realistic representation of nidus vasculature and complexity derived from patients. The injection of liquid embolic material performed in this model replicated real-life treatment conditions. The plug and push technique was successfully simulated to penetratreliquid embolic material into the AVM nidus. The flow data from 4D flow MRI were comparable to CFD analysis.

Conclusions An in vitro human brain AVM model with realistic complexities of nidus was successfully manufactured using $3 \mathrm{D}$ printing technology. The model demonstrated realistic pliability during the liquid embolic material injection and also feasibility of flow analysis. This in vitro AVM model may represent a valuable tool for simulation, flow research and development of new materials or technique.

Disclosures N. Kaneko: None. H. Ullman: None. F. Ali: None. P. Berg: 1; C; German Research Foundation, Federal Ministry of Education and Research within the Forschungscampus STIMULATE. Y. Ooi: None. S. Tateshima: 2; C; Cerenovus, Medtronic, Stryker. G. Colby: 2; C; Medtronic, Microvention, Stryker. Y. Komuro: None. P. Hu: None. V. Szeder: None. M. Nour: None. L. Guo: None. A. Chien: None. F. Vinuela: None. S. Nemoto: None. J. Hinman: None. G. Duckwiler: 1; C; Tarsadia Foundation. 2; C; Medtronic. R. Jahan: None.

\section{E-037 ROBOTIC TRANSCRANIAL DOPPLER USE ANEURYSMAL SUBARACHNOID HEMORRHAGE: A SAFETY AND EFFICACY STUDY}

${ }^{1} \mathrm{~K}$ Clare $*{ }^{2} \mathrm{~A}$ Stein, ${ }^{2} \mathrm{~J}$ Cooper, ${ }^{2} \mathrm{C}$ Gandhi, ${ }^{2} \mathrm{C}$ Bowers, ${ }^{2} \mathrm{C}$ Cole, ${ }^{2} \mathrm{~J}$ Santarelli, ${ }^{2} \mathrm{~J}$ Pisapia, ${ }^{2} \mathrm{~F}$ Al-Mufti. ${ }^{1} \mathrm{New}$ York Medical College, Valhalla, NY; ${ }^{2}$ Neurology and Neurosurgery, Westchester Medical Center, Valhalla, NY

\subsection{6/neurintsurg-2020-SNIS.73}

Introduction/Purpose Transcranial doppler (TCD) is a bedside modality to rapidly assess the presence of vasospasm or stenosis of cerebral arteries. However, manual TCD requires a trained sonographer which may not be available in all healthcare settings. The LUCID TCD system aims to expedite and enhance TCD blood flow measurements through semi-autonomous acquisition. This study aims to evaluate the safety and feasibility of the LUCID TCD system for detection of vasospasm.

Materials and Methods Peak velocities of the middle cerebral arteries (MCA) were measured and a velocity greater than $120 \mathrm{~cm} / \mathrm{s}$ was considered indicative of vasospasm. To determine feasibility, maximal MCA velocities from LUCID TCD were compared to computer tomography angiography, and the Cohen's Kappa value was calculated to gauge the agreement 